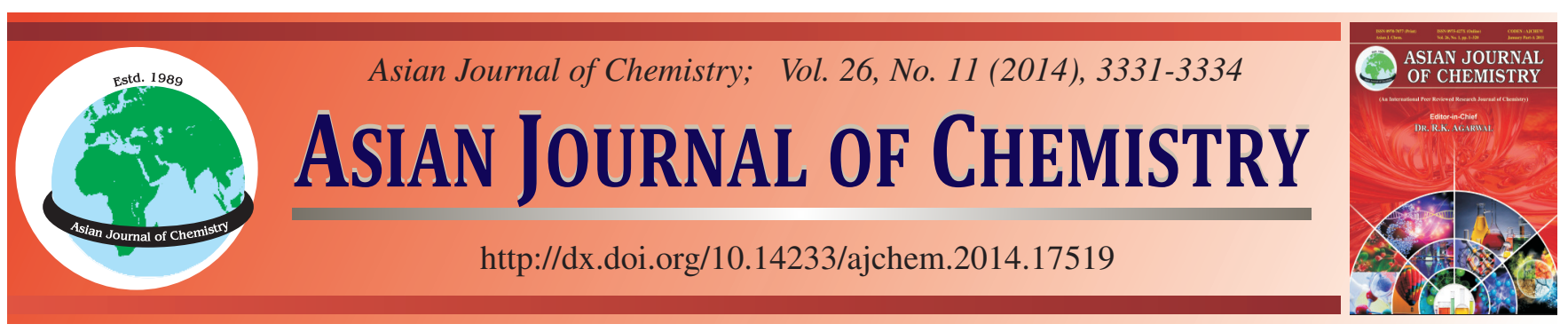

\title{
Isolation and Purification of Gingerols from Ginger by High-Speed Counter-Current Chromatography
}

\author{
Shirong TANG*, Hui Song, ENQI LiU and JiAnting QI
}

School of Food Engineering, Xuzhou Institute of Technology, Xuzhou 2211111, Jiangsu Province, P.R. China

*Corresponding author: E-mail: tangsr@163.com

Received: 10 February 2014;

Accepted: 14 April 2014;

Published online: 25 May 2014;

AJC-15242

\begin{abstract}
High-speed counter-current chromatography (HSCCC) was successfully used for isolation and purification of 6-gingerol, 6-shogaol, 8-gingerol and10-gingerol from the crude ethanol extract of ginger by a consequently stepwise elution which was performed by eluting with the lower phase of $n$-hexane-ethyl acetate-methanol-water (5.5:5:5.5:5) first and then eluting with the lower phase of $n$-hexane-ethyl acetate-methanol-water (7:5:7:5), when the upper phase of $n$-hexane-ethyl acetate-methanol-water (5.5:5:5.5:5) was used as the stationary phase in the head to tail elution mode. The preparative HSCCC separation was performed on $500 \mathrm{mg}$ of the crude extract yielding pure 6-gingerol (12 mg), 6-shogaol (2 mg), 8-gingerol (4 mg) and 10-gingerol (4 mg) at purities of 92.7, 96.8, 91.2 and $92.3 \%$, respectively, as determined by high performance liquid chromatography (HPLC). HSCCC is a powerful technique for isolation and separation of chemical composition from ginger.
\end{abstract}

Keywords: High-speed counter-current chromatography, Isolation, Gingerol, Shogaol, Ginger.

\section{INTRODUCTION}

Ginger (Zingiber officinale Rosc) has been used in foods as a spice around the world for centuries ${ }^{1}$ and it had been widely used as a traditional medicine for relief of nausea or inflammation associated with arthritis ${ }^{2}$. Recently, ginger has received extensive attention as a botanical dietary supplement in the USA and Europe because of its antiplatelet aggregation, anticancer antioxidative ${ }^{3}$, antiinflammatory and antitumor activities ${ }^{4}$, etc. The major bioactive constituents in ginger were the so-called "pungent principles", the gingerols 5 . The gingerols, a series of homologues differentiated by the length of their unbranched alkyl chains, were identified as the major pungent components in the ginger oleoresin from fresh rhizome, of which 6-gingerol is the most abundant. And gingerol can be converted into shogaol, zingerone and paradol. Shogaols are found in small amounts in fresh ginger and in large amounts in stored products $^{6} .6$-Shogaol is an anhydride of 6-gingerol. 6-gingerol and 6-shogaol have been shown to have a number of pharmacological activities including antipyretic, analgesic, antitussive and hypotensive effects ${ }^{7-9}$. Further studies to better understand the biochemical properties are needed to ascertain their clinical applications and this necessitates the development of efficient, parative-scale separation methods for their isolation and purification ${ }^{10}$. Moreover, the preparation of active compounds or fractions as reference standards for quality control purposes is also in urgent need in research as well as industrial communities. However, most ginger preparations are mixtures of multiple components. The conventional methods including solvent extraction, column chromatographic separation with silica gel and/or polyamide are difficult to separate these compounds, respectively from ginger in semi-preparative scale due to irreversible adsorptions of separation materials onto the solid support during separation. Hence, alternative method has gained growing importance.

High-speed counter-current chromatography (HSCCC) is a form of liquid-liquid partition chromatography ${ }^{11}$. Solute separation is based on partitioning between the two immiscible liquid phases: the mobile phase and the support-free liquid stationary phase. Without any solid matrix, the stationary phase is retained in the column by centrifugal force ${ }^{12}$. When the mobile phase is pumped through the column, sample components are partitioned between the two phases and they are separated on the basis of difference in partition coefficients. Comparing with traditional liquid-solid column chromatography, HSCCC eliminates irreversible adsorption of samples onto the solid support and yields higher recovery and efficiency. So it is considered as a suitable alternative for separation of active 
components from traditional Chinese medicinal herbs and other natural products. Many successful applications of HSCCC have been reported for the separation of various components such as alkaloids ${ }^{13}$, flavonoids ${ }^{14}$, polyphenols ${ }^{15}$, etc.

In view of the wide biologically activities, the preparation of gingerols with high purity has been of much interest to pharmaceutical chemists. To obtain pure compounds by conventional separation methods. In this paper, the development of HSCCC method for the separation and purification of gingerols from the crude methanol extract of ginger will be described. The optimum conditions were obtained, which led to the successful separation of gingerols with the purity of each at over $95 \%$.

\section{EXPERIMENTAL}

The HSCCC instrument employed in the present study is TBE-300B high-speed counter-current chromatography (Tauto Biotechnique Company, Shanghai, China) with three multilayer coil separation column connected in series (I.D. of the tubing $=1.6 \mathrm{~mm}$, total volume $=260 \mathrm{~mL}$ ) and a $20 \mathrm{~mL}$ sample loop. The revolution radius or the distance between the holder axis and central axis of the centrifuge (R) was $5 \mathrm{~cm}$ and the $\beta$ values of the multilayer coil varied from 0.5 at internal terminal to 0.8 at the external terminal $(\beta=r / R$, where $r$ is the distance from the coil to the holder shaft). The revolution speed of the apparatus can be regulated with a speed controller in the range between 0 and $1000 \mathrm{rpm}$. An HX 1050 constant-temperature circulating implement (Beijing Boyikang Lab Instrument Company, Beijing, China) was used to control the separation temperature. A $\triangle \mathrm{KTA}$ prime system (Amersham Pharmacia Biotechnique Group, Sweden) was used to pump the two-phase solvent system and perform the UV absorbance measurement. It contains a switch valve and a mixer, which were used for gradient formation. The data were collected with Sepu 3000 chromatography workstation (Hangzhou Puhui Science Apparatus Company, Hangzhou, China). The HPLC equipment used was Agilent 1100 HPLC system including G1311A QuatPump, G1313A autosampler and G1314A variable wavelength detector, G1332 degasser and Agilent HPLC workstation.

All solvents used for preparation of crude sample and HSCCC separation were of analytical grade, methanol used for HPLC was chromatographic grade. Both were purchased from Sinopharm group pharmaceutical Co. Ltd. (Shanghai, China). 6-gingerol, 6-shogaol, 8-gingerol and 10-gingerol were purchased from National Institute for the Control of Pharmaceutical and Biological Products, Ministry of Health (Beijing, China). Water used as the mobile phase in HPLC was prepared with Millipore purifier (Millipore, USA) in our laboratory.

Preparation of crude sample: Preparation of crude sample was carried out as following. The dried fruits of ginger were ground to powder (about $60 \mathrm{mesh}$ ). The powder (100 g) was dipped in $500 \mathrm{~mL}$ of $60 \%$ ethanol for $0.5 \mathrm{~h}$ and then extracted by sonication using a SK3200LH ultrasonic cleaning instrument (Shanghai Kudos Ultrasonic Instrument Co., Shanghai, China) for $15 \mathrm{~min}$. The extraction procedure was repeated twice. The extracts were combined and concentrated under reduced pressure with a Model SENCO R-206 rotary evaporator (Shanghai Shensheng Biotech Co., Shanghai, China). $27.6 \mathrm{~g}$ of crude extract powder was obtained. It was stored in a refrigerator $\left(4^{\circ} \mathrm{C}\right)$ for the subsequent HSCCC separation.
Selection of two-phase solvent system: A number of twophase solvent systems were tested by changing the volume ratio of the solvent to obtain the optimum composition that gave suitable partition coefficient $(\mathrm{K})$ values of the target compounds. The K-values were determined by HPLC as following: $0.5 \mathrm{mg}$ of crude sample was added to a test tube, to which $5 \mathrm{~mL}$ of each phase of the two-phase solvent system was added. The test tube was shaken violently for several minutes, then was analyzed by HPLC to obtain the partition coefficients (K). Then upper and lower phase were analyzed by HPLC with equal volume. The $\mathrm{K}$ value was expressed as the peak area of the compound in the upper phase divided by the peak area of the same compound in the lower phase.

Preparation of two-phase solvent system and sample solution: Two-phase solvent system utilized in the HSCCC separation and purification was prepared by mixing $n$-hexane, ethyl acetate, methanol and water and then thoroughly equilibrating the mixtures in a separator funnel at room temperature. The upper phase and the lower phase were separated and degassed by sonication for $0.5 \mathrm{~h}$ prior to use. The volume ratios of the four solvents are 5:5:5:5 and 7:5:7:5, used, respectively for the separation and purification runs.

The sample solution was prepared by dissolving the crude extract sample in the mixture of $10 \mathrm{~mL}$ of the upper phase and $10 \mathrm{~mL}$ of the lower phase of the solvent system.

HSCCC separation procedure: The multi-layer coiled column was first filled completely with the upper solvent phase (stationary phase). Then the apparatus was rotated at $850 \mathrm{rpm}$, while the lower phase was pumped into the head of the column at a flow rate of $2 \mathrm{~mL} / \mathrm{min}$. All through the experiment, the separation temperature was controlled at $25^{\circ} \mathrm{C}$. After the mobile phase front emerged and hydrodynamic equilibrium was established in the column, the sample solution was injected through the sample injection valve. The column effluent was monitored with a UV detector at $280 \mathrm{~nm}$ and each peak fraction was collected manually according to the chromatographic profile displayed on the recorder and evaporated under reduced pressure. The residual was dissolved in methanol for subsequent study. The retention of the stationary phase relative to the total column capacity was computed from the volume of the stationary phase collected from the column after the separation was completed.

HPLC analysis of HSCCC peak fractions: The crude extract of ginger and each peak fraction from HSCCC separation were analyzed by HPLC. The column used for HPLC analysis was Hypersil $\mathrm{C}_{18}$ column $(250 \mathrm{~mm} \times 4.6 \mathrm{~mm}$ i.d. 5 $\mu \mathrm{m})$ (Dalian Elite Analytical Instruments Co. Ltd.). The mobile phase was acetonitrile $(\mathrm{A})$ and water $(\mathrm{B})$ with gradient elution, gradient program: $60 \% \mathrm{~B}, 0-25 \mathrm{~min} ; 60 \% \mathrm{~B}$ to $35 \% \mathrm{~B}, 25-$ $35 \mathrm{~min} ; 35 \% \mathrm{~B}$ to $60 \% \mathrm{~B}, 35-40 \mathrm{~min}$. The mobile phase was eluted at a flow rate of $1.0 \mathrm{~mL} / \mathrm{min}$ and the effluent was monitored at $280 \mathrm{~nm}$.

\section{RESULTS AND DISCUSSION}

Optimization of HPLC method: The partition coefficient of each component in crude extract was determined by HPLC analysis. So in the first place, a good HPLC method should be developed for analysis of crude extract. Different mobile phases (methanol-water, acetonitrile-water) and different elution 
modes were used in HPLC to separate ginger polyphenols from the crude extract. The results indicated that when acetonitrile (A) and water (B) was used as the mobile phase with gradient elution, a satisfactory separation of targeted compounds was obtained $^{16}$. The crude sample and peak fractions separated by HSCCC were analyzed by HPLC under the optimum conditions. The purity factor of each peak was within the calculated threshold limit. The chromatograms of crude extract were shown in Fig. 1(A).

Optimization of HSCCC conditions: In a HSCCC experiment, selection of the two-phase solvent system is the first and critical step; a good solvent system can provide an ideal partition coefficient $(\mathrm{K})$ for the target compounds. The key of solvent optimization is first to find a solvent combination in which the sample is freely soluble, then to adjust this solvent combination to ensure that the $\mathrm{K}$ value of the target compounds is close to 1 . The $\mathrm{K}$ value of a two-phase solvent system is critical for efficient separation. If it is much smaller than 1 , the solutes will be eluted close to each other near the solvent front, which may result in loss of peak; if the $\mathrm{K}$ value is much greater than 1 , the solutes will be eluted in excessively broad peaks and may lead to extended elution time. In order to achieve efficient resolution of target compounds in HSCCC separation, the Kvalues of these two compounds in different solvent systems were determined by HPLC ${ }^{17}$.

Ginger polyphenols were difficult to separate because of their closely related structure and property. A series of twosolvent system of $n$-hexane-ethyl acetate-methanol-water were tested for estimation the $\mathrm{K}$-values. The results are given in Table-1.

It can be seen from Table-1 that the K-values were more than 1 when the solvent system of $n$-hexane-ethyl acetatemethanol-water (5:5:5:5) was tested first and addition of methanol and $n$-hexane to the solvent system could improve the K-values. When $n$-hexane-ethyl acetate-methanol-water (5.5:5:5.5:5) was used as the two-phase solvent system, the $\mathrm{K}$ values were suitable for 6-gingerol, 6-shogaol and 8-gingerol, the $\mathrm{K}$ values was a little high for 10-gingerol. When $n$-hexaneethyl acetate-methanol-water (7:5:7:5) was used as the twophase solvent system, the K value was suitable for 10-gingerol. Thus, when the upper phase of solvent system composed of $n$-hexane-ethyl acetate-methanol-water (5.5:5:5.5:5) was used as the stationary phase, 6-gingerol, 6-shogaol, 8-gingerol and 10 -gingerol could be eluted out at a desired time by a consequently stepwise elution which was performed by eluting with the lower phase of $n$-hexane-ethyl acetate-methanol-water (5.5:5:5.5:5) first and then eluting with the lower phase of $n$ hexane-ethyl acetate-methanol-water $(7: 5: 7: 5)^{18}$.
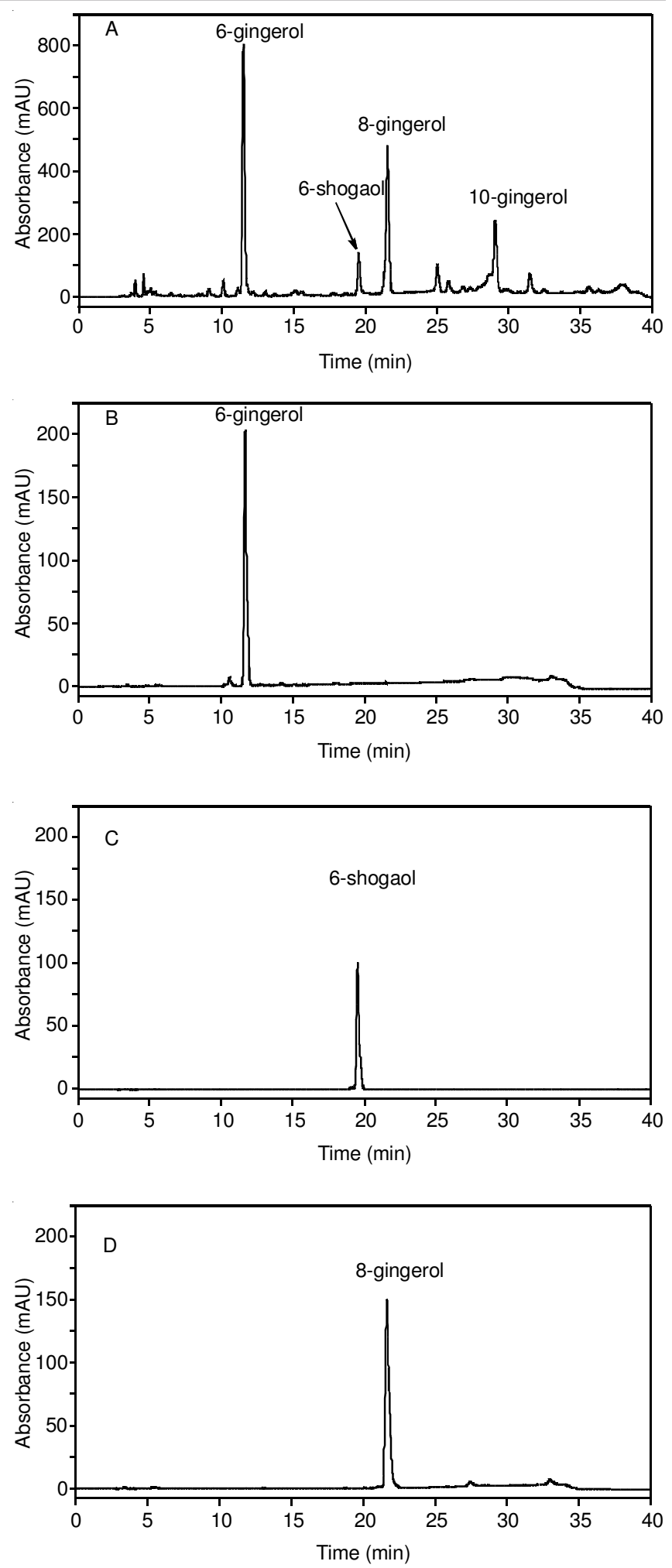

TABLE-1

K VALUES OF GINGER POLYPHENOLS IN DIFFERENT SOLVENT SYSTEMS

Solvent system ( $n$-hexane-ethyl acetate-methanol-water) $(\mathrm{v} / \mathrm{v})$

$5: 5: 5: 5$

$5.5: 5: 5.5: 5$

$6: 5: 6: 5$

$6.5: 5: 6.5: 5$

$7: 5: 7: 5$

7.5:5:7.5:5

$\begin{array}{cc} & \\ \text { 6-Gingerol } & 6 \\ 0.2573 & \\ 0.8884 & \\ 0.4455 & \\ 0.2653 & \\ 0.1889 & \\ 0.1226 & \end{array}$

$\mathrm{K}$ value

\begin{tabular}{ccc} 
6-Shogaol & 8-Gingerol & 10-Gingerol \\
\hline 2.5402 & 3.2402 & 15.0747 \\
1.1941 & 1.5854 & 10.5133 \\
0.8269 & 1.0275 & 4.7041 \\
0.5714 & 0.8462 & 1.7001 \\
0.4204 & 0.5649 & 1.2147 \\
0.2453 & 0.3493 & 0.7234 \\
\hline
\end{tabular}




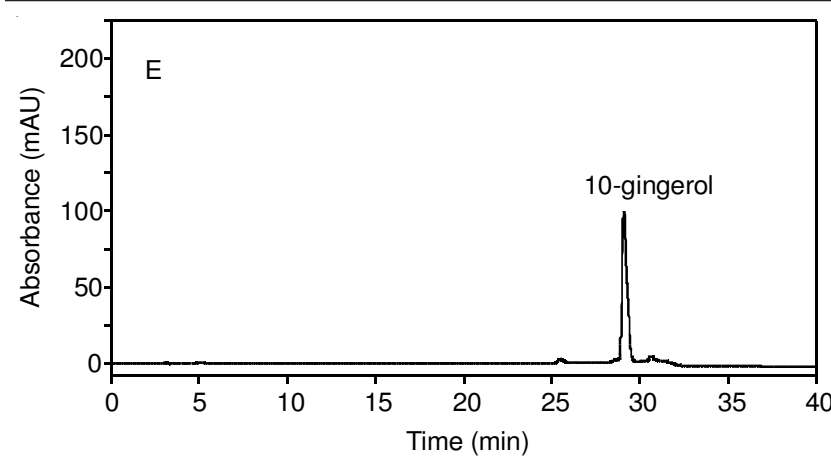

Fig. 1. HPLC chromatograms of crude extract from ginger and HSCCC peak fractions. Conditions: column, Hypersil $\mathrm{C}_{18}$ column $(250 \mathrm{~mm}$ $\times 4.6 \mathrm{~mm}$ i.d. $5 \mu \mathrm{m}$ ); mobile phase, acetonitrile (A) and water (B) with gradient elution, gradient program: $60 \% \mathrm{~B}, 0-25 \mathrm{~min} ; 60 \% \mathrm{~B}$ to $35 \% \mathrm{~B}, 25-35 \mathrm{~min} ; 35 \% \mathrm{~B}$ to $60 \% \mathrm{~B}, 35-40 \mathrm{~min}$; flow rate, 1.0 $\mathrm{mL} \min ^{-1}$; detection wavelength, $280 \mathrm{~nm}$. (A) crude ethanol extract from ginger; (B) peak I; (C) peak II; (D) peak I; (E) peak II of Fig. 2

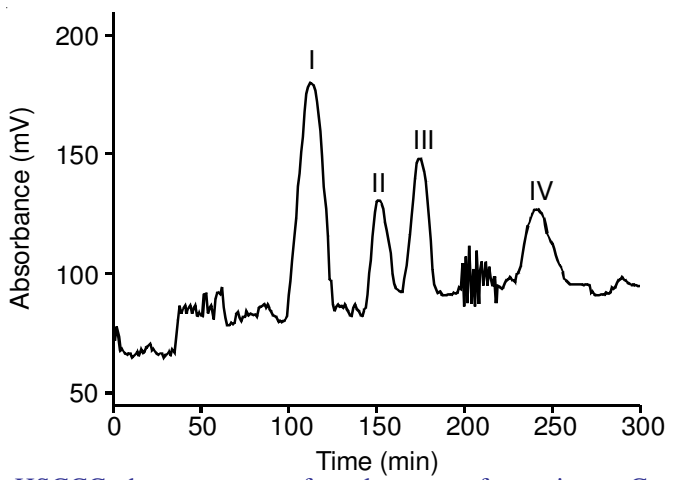

Fig. 2. HSCCC chromatogram of crude extract from ginger. Conditions: Stationary phase: the upper phase of the $n$-hexane-ethyl acetatemethanol-water $(5.5: 5: 5.5: 5, \mathrm{v} / \mathrm{v})$; stepwise elution: lower phase of $n$-hexane-ethyl acetate-methanol- water $(5.5: 5: 5.5: 5, \mathrm{v} / \mathrm{v})$ for the first $3 \mathrm{~h}$ and lower phase of the $n$-hexane-ethyl acetate-methanol-water $(7: 5: 7: 5, \mathrm{v} / \mathrm{v})$ for the second $2 \mathrm{~h}$; flow rate, $2.0 \mathrm{~mL} \mathrm{~min}^{-1}$; revolution speed, $800 \mathrm{rpm}$; detection wavelength, $280 \mathrm{~nm}$; separation temperature, $25^{\circ} \mathrm{C}$; sample size, $500 \mathrm{mg}$ of crude sample dissolved in mixture of $10 \mathrm{~mL}$ of the upper phase and $10 \mathrm{~mL}$ of the lower phase; retention percentage of the stationary phase, $54.5 \%$. I: 6gingerol (collected during 100-130 min); II: 6-shogaol (collected during 145-160 $\mathrm{min}$ ); III: 8-gingerol (collected during 165-180 min); IV: 10-gingerol (collected during 230-260 min)

When chloroform-methanol-water at the ratio of 4:3:2 was used as the two-phase solvent system, the target compounds mainly partitioned in the lower phase. The K-values were very small. Target compounds were eluted together closely near the solvent front. Addition of methanol and reduction of water to the solvent system could improve the K-values, but the Kvalues were more than 1 . So, chloroform-methanol-water was unsuitable for separation of ginger polyphenols.

The crude sample $(500 \mathrm{mg})$ was dissolved in the mixture of $10 \mathrm{~mL}$ of the upper phase and $10 \mathrm{~mL}$ of the lower phase of the solvent system of $n$-hexane-ethyl acetate-methanol-water (5.5:5:5.5:5). The sample solution was separated and purified by HSCCC according to the procedure described above. The upper phase of $n$-hexane-ethyl acetate-methanol-water (5.5:5:5.5:5) was used as the stationary phase while the lower phase was used as the mobile phase in the head to tail elution mode by a consequently stepwise elution.

Other conditions such as the revolution speed of the separation column, the flow rate of the mobile phase and the temperature were also investigated. When the flow rate of 2.0 $\mathrm{mL} \mathrm{min}^{-1}$, revolution speed of $800 \mathrm{rpm}$ and the separation temperature of $25^{\circ} \mathrm{C}$ were employed in HSCCC separation, the retention percentage of the stationary phase could be kept at $54.5 \%$. The crude samples from ginger were separated and purified under the optimum HSCCC conditions. The typical HSCCC chromatogram was shown in Fig. 2. 6-Gingerol, 6shogaol, 8-gingerol and 10-gingerol were obtained by a consequently stepwise elution and yielded $12 \mathrm{mg}$ of 6-gingerol(I), $2 \mathrm{mg}$ of 6-shogaol(II), $4 \mathrm{mg}$ of 8-gingerol(III) and $4 \mathrm{mg}$ of 10-gingerol(IV) from $500 \mathrm{mg}$ of crude sample. The four substances were further identified by HPLC.

HPLC analysis of HSCCC peak fractions: The purity of each peak fraction of HSCCC was determined by HPLC. The HPLC chromatograms of each fraction were shown in Fig. 1(BE). The purity of 6-gingerol(I), 6-shogaol(II), 8-gingerol(III) and 10-gingerol(IV) was 92.7,96.8,91.2 and $92.3 \%$, respectively.

\section{Conclusion}

HSCCC has been developed and successfully applied to the separation and purification of 6-gingerol, 6-shogaol, 8gingerol and 10-gingerol in crude extract of ginger. The results illustrate that HSCCC is an effective methodology for selective semi-preparative scale separation and purification of bioactive components in herbal extracts.

\section{ACKNOWLEDGEMENTS}

This work was financially sponsored by Qing Lan Project and National Spark-plan (2011GA690046).

\section{REFERENCES}

1. J.S. Chang, K.C. Wang, C.F. Yeh, D.E. Shieh and L.C. Chiang, J. Ethnopharmacol., 145, 146 (2013).

2. F. Peng, Q.F. Tao, X.M. Wu, H. Dou, S. Spencer, C. Mang, L. Xu, L. Sun, Y. Zhao, H. Li, S. Zeng, G. Liu and X. Hao, Fitoterapia, 83, 568 (2012).

3. G. Oboh, A.J. Akinyemi and A.O. Ademiluyi, Exp. Toxicol. Pathol., 64, 31 (2012).

4. M.S. Ding, M. Leach and H. Bradley, Women Birth, 26, 26 (2013).

5. N. Pawar, S. Pai, M. Nimbalkar and G. Dixit, Food Chem., 126, 1330 (2011).

6. B.H. Ali, G. Blunden, M.O. Tanira and A. Nemmar, Food Chem. Toxicol., 46, 409 (2008).

7. M.L.B. Ahui, P. Champy, A. Ramadan, L. Pham Van, L. Araujo, K. Brou André, S. Diem, D. Damotte, S. Kati-Coulibaly, M.A. Offoumou, M. Dy, N. Thieblemont and A. Herbelin, Int. Immunopharmacol., 8, 1626 (2008).

8. K.R. Shanmugam, K. Mallikarjuna, N. Kesireddy and K. Sathyavelu Reddy, Food Chem. Toxicol., 49, 893 (2011).

9. K.R. Shanmugam, K. Mallikarjuna, K. Nishanth, C.H. Kuo and K.S. Reddy, Food Chem., 124, 1436 (2011).

10. X.W. Huang, L.J. Chen, Y.B. Luo, H.Y. Guo and F.Z. Ren, J. Dairy Sci., 94, 2259 (2011).

11. Y. Ito, J. Biochem. Biophys. Methods, 5, 105 (1981).

12. E. Yuan, B.G. Liu, Z.X. Ning and C. Chen, Food Chem., 115, 1158 (2009).

13. J.L. Liang, Z. Yang, X.J. Cao, B. Wu and S. Wu, J. Chromatogr. A, 1218, 6191 (2011).

14. X.P. Dai, Q. Huang, B.T. Zhou, Z. Gong, Z. Liu and S. Shi, Food Chem., 139, 563 (2013).

15. Q.H. Chen, M.L. Fu, M.M. Chen, J. Liu, X. Liu, G. He and S. Pu, Food Chem., 132, 619 (2012).

16. C.N.A. Salmon, Y.A. Bailey-Shaw, S. Hibbert, C. Green, A.M. Smith and L.A.D. Williams, Food Chem., 131, 1517 (2012).

17. X. Wang, Z.J. Zheng, X.F. Guo, J. Yuan and C. Zheng, Food Chem., 125, 1476 (2011).

18. Q.L. Qiao and Q.Z. Du, J. Chromatogr. A, 1218, 6187 (2011). 